CLAWAR 2018: 21st International Conference on Climbing and Walking Robots and the Support Technologies for Mobile Machines, Panama City, Panama, 10-12 September 2018

\title{
DESIGN OF A SYSTEM TO SUPPORT THE MOBILITY OF VISUALLY IMPAIRED PEOPLE*
}

\author{
HECTOR MONTES ${ }^{1,2}$, IGNACIO CHANG ${ }^{1}$, GUELDA CARBALLEDA ${ }^{3}$, JOSE \\ MUÑOZ $^{1}$, ANTONY GARCIA ${ }^{1}$, RAFAEL VEJARANO ${ }^{3}$, YESSICA SAEZ ${ }^{1}$ \\ ${ }^{I}$ Faculty of Electrical Engineering, Universidad Tecnológica de Panamá, Panama; \\ ${ }^{2}$ Field and Service Robotics Group, Centre for Automation and Robotics (CSIC-UPM); \\ ${ }^{3}$ Faculty of Systems Engineering and Computer Science, Universidad Tecnológica de \\ Panamá, Panama, hector.montes1@utp.ac.pa
}

\begin{abstract}
The autonomous mobility for visually impaired people has been an issue in where several researchers have contributed with proposals in order to solve it. However, there is a lot of work to carry out in the cities in where this kind of technology has been applied, but also in other cities or town where this or other technology not has arrived yet. In this work two different ICT-based systems have been described, which provide helps in the mobility of visually impaired people in the public transport in Panama. One of them consists of the development of new applications for Android-based smartphones, and the second system is based on the development of RF modules into the ISM radio band. These prototypes are used by the visually impaired people and by the bus driver, and one of them is installed at the bus stop.

Keywords: Visually impaired people; autonomous mobility; RF modules; Android-based applications; public transportation system.
\end{abstract}

\section{Introduction}

Visual impairment can limit people's ability to perform everyday tasks and can affect their quality of life and ability to interact with the surrounding world [1]. In addition, blindness, the most severe form of visual impairment, can reduce people's ability to perform daily tasks, and move about unaided. Good quality rehabilitation allows people with different degrees of visual impairment to fully profit from life, achieve their goals and be active and productive in today's society [1]. However, in a parallel effort, it is possible to use the technology to support the mobility of these people at indoor and outdoor locations.

The World Health Organization (WHO) in the Fact Sheet Updated October 2017 establishes that they count with a global action plan 2014-2019, approved by the World Health Assembly in 2013, with the aim of achieving a measurable

\footnotetext{
* This work is supported by grant 109-2015-4-FID14-073 (MOVIDIS project) of the SENACYT of Panama.
} 
reduction of $25 \%$ of avoidable visual impairments by 2019 [2]. However, this declaration asserts there is a problem and must be attended.

In [2] is estimated the following: (i) 253 million people live with vision impairment: 36 million are blind and 217 million have moderate to severe vision impairment, (ii) over $80 \%$ of all vision impairment can be prevented or cured. However, most people visually impaired live in low-income settings.

In order to support or help in the mobility of people with visual disability (in several environments), some research groups have carried out different proposals based on technology, e.g., the typical canes with an ultrasound system, laser, or any other similar technology, which informs the user if an obstacle has been detected by means of different types of signs. However, these systems have had few followers, since they frequently required training for the visually impaired people (ViIP) and, besides, they forced to keep more attention on the signals of the device than on the user's own perception of the route they were taking [3].

As mentioned above, there are several works and lines of research based on ICT that are being developed in various universities and research centers, to provide aids in the mobility and orientation of the ViIP. Some works of interest can be reviewed in [4-10]. These works include mobility in public passenger transport, the use of the smartphones, other electronic mobile devices. All of them are currently under development.

In this article, a new design of a dual system to support the mobility of visually impaired people in public transport system in Panama is described. The dual system consists of a low-cost RF system and new applications for smartphone Android-based. In both systems, the main goal is to provide aids based on ICT for that the visually impaired people, in an autonomous way, are able to use the public transport system in Panamá [11-13].

The rest of the article is organised as follow: in Section 2 is described the design of both systems, the apps, and the RF sub-system. Afterward, Section 3 presents some technical evaluations of the system. Finally, conclusions and future works are given in Section 4.

\section{Design description of the system}

This work has been carried out within the framework of the MOVIDIS project (http://movidis.utp.ac.pa/) [11], funded by the SENACYT of Panama (http://www.senacyt.gob.pa/). This project consists of the design and development of two different prototypes with the aim to provide assistance for the autonomous mobility of ViIP in public transportation system in Panama. One of the prototypes is related to the design of new apps for Android-based smartphones 
for ViIP, her/his supervisor (if available), and bus driver. The other prototype consists of a set of RF subsystems that interact among them and localized with the ViIP, the bus, and the bus stop.

\subsection{Design of the Apps}

Three apps with their different modified versions have been designed and developed for the visually impaired people (ViDis app), the bus driver (ViBus app) and the ViIP supervisor (Tutor app). In Figure 1 is presented the functioning scheme of the designed apps with the server, which has a specific database for this project. PHP (Hypertext Pre-processor) is a powerful and widely-used open source server-side scripting language to write dynamically generated web pages.

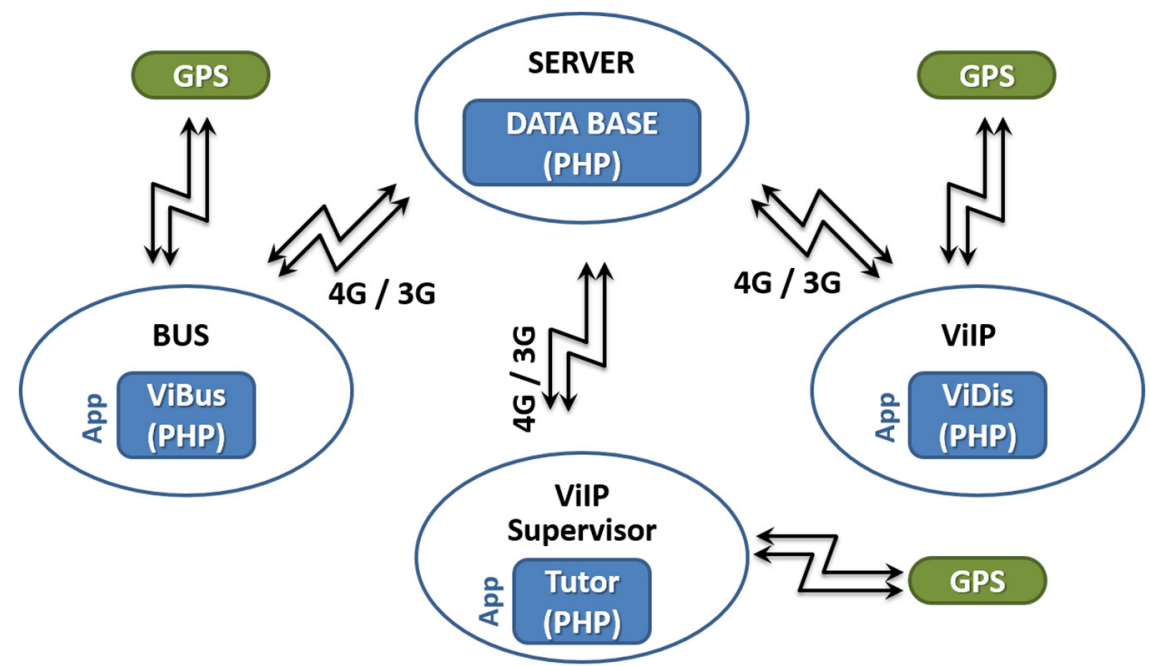

Figure 1. Functioning scheme of the apps.

In this article, the functioning of the system between the ViDis and ViBus apps will be described. The interaction between both apps can be performed when each app is activated in its smartphone. Besides, must be considered the ViIP and bus driver have been trained in the use of each app. The protocol of actuation between the ViIP and the driver, beginning when the ViIP request the bus stop up to him/her get off the bus is described as follow:

- The bus is on the route and the ViIP is on the bus stop.

- The ViIP is able to know, through the ViDis app, the bus is near to bus stop. Therefore, the ViIP request the bus stop, and he/she is waiting the bus answer. 
- The ViBus app detects the bus stop request, and the driver touching the phone screen to send the received message.

- The ViIP receives the bus answer by means of vibration and/or audible message. With this, the first interaction is realized.

- When the ViIP is on the bus, she/he can recognize in advance the different bus stop along the route, by means of the ViDis app. Therefore, the ViIP can request the bus stop at any moment using the app.

- Immediately after request the bus stop to get off on the part of ViIP, the ViBus app receive it. Then, the bus drive send a positive answer to the ViIP touching his phone screen.

- The ViIP receives this answer by means of vibrations and/or audible message. With this, the second protocol interaction is realized.

\subsection{Design of the RF System}

Three RF modules have been designed and developed for the visually impaired people (MOVI-ETA), the bus driver (MOVI-Bus) and the bus stop (MOVI-Stop). These modules transmit their RF signals in the ISM radio band since this radio band is reserved internationally for industrial, scientific and medical purposes other than commercial telecommunications [14] and, besides, it is license-free. In Figure 2 is presented the interaction scheme among the designed RF modules for the MOVIDIS project.

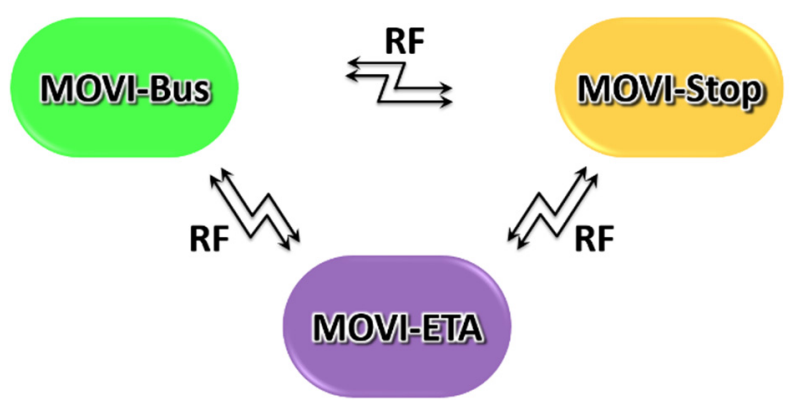

Figure 2. Interaction scheme among the modules of RF system to 433.4-473.0 MHz's

The general functioning of this RF system is as follow:

- A bus is on the route and it is emitting specific RF signal by means of the MOVI-Bus module with the aim to detect the MOVI-Stop modules.

- When the MOVI-Bus detect any MOVI-Stop, the first recognize the bus stop where the second is installed. 
- At this moment, if there is a VoIP using MOVI-ETA at that bus stop, the MOVI-Stop detect it and sends a message to the MOVI-ETA of recognizing, and the link is established between them.

- If the ViIP requires stopping the bus, then he/she touch a button of MOVI-ETA and request the bus stop through the MOVI-Stop.

- Afterward, the bus driver send a message to the MOVI-ETA trough the MOVI-Stop indicating that will stop the bus. With this, the first interaction protocol finalise.

- When the ViIP is inside the bus, the MOVI-ETA will connect with the MOVI-Bus. Then, the MOVI-ETA will recognise all bus stop along the route through the MOVI-Bus, since it is will connect with each MOVIStop.

- Therefore, the VilP can request using the MOVI-ETA the bus stop to get off. The bus driver send a message of received. With this, the second interaction protocol finalise.

Both interaction protocols, using apps for smartphones or RF modules using ISM radio band are summarized in this paper (but sufficient for understanding them), since different signals, flags, and process are executed in each moment, however, its description would be the paper extensive.

Why does it necessary to design and developed two different systems to provide help for the ViIP in their mobility in the public transportation of Panama? Because there is no GPS connection and $3 \mathrm{G} / 4 \mathrm{G}$ services in several routes in different cities in Panama. Other reasons are that in some zones in Panama City, some places, bus stops, etc., there are not labelled, and neither at other cities of the country. Therefore, the two systems will be very useful in the future. Besides, this problem is not only of Panama, but also of other countries of the region and other towns in developed countries. Consequently, this project can be useful for improve the mobility of the ViIP in the public transport in general.

\section{Evaluation of the systems}

Firstly, the visually impaired people that collaborate with the MOVIDIS project have been trained with the two systems, the ViDis app, and the MOVI-ETA module. Figure 3 shows the snapshot of the training process. Both collaborators have a full visual disability and the training process have taken a session less than 30 minutes. This demonstrates the phone app and the RF module have userfriendly interface design. In addition, the bus drivers have been trained with the ViBus app and the MOVI-Bus module. 
Several experimental tests have been carried out in order to evaluate the designed prototypes in lab conditions and in outdoor conditions using different types of motor vehicles. Figure 4 and Figure 5 show two different scenes when a ViIP is inside the bus and when she is waiting for the bus using the ViDis app. Both experiments have been carried out successfully, shown promising results.

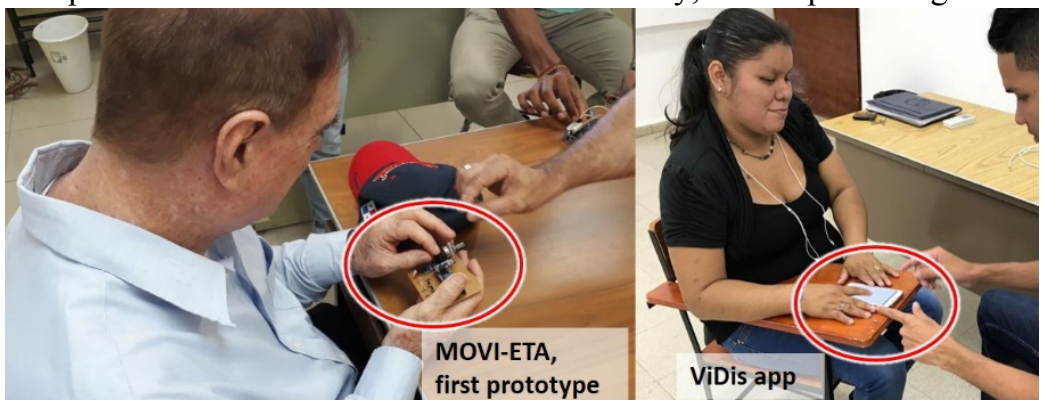

Figure 3. ViIP training process with the MOVI-ETA prototype and the ViDis app.

In the experiment, represented in Figure 4, both app knew the next bus stop, so the ViIP is able to request the bus stop at the respective moment. The bus driver receives this request and sends the response to the ViIP and, subsequently, stop the bus.
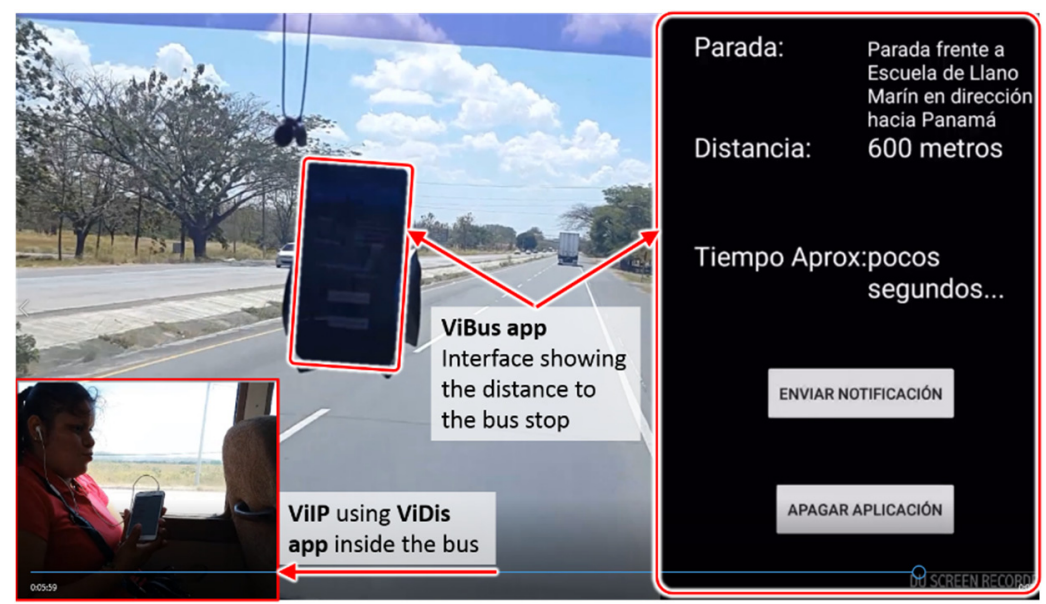

Figure 4. ViIP inside the bus using the ViDis app and the ViBus interface, during an experimental test.

In Figure 5 is possible to see the user-friendly interface design of the ViDis app. This interface has big buttons, which send her vibration and audible signals when she touches them. Besides, she receives vibration signals and audible 
messages when the bus driver sends some responses to her. Therefore, the ViIP is aware of the interaction with the bus, before and after taking it. The interaction time of the bus driver with de ViBus app is negligible; therefore, it is not compromised road safety.

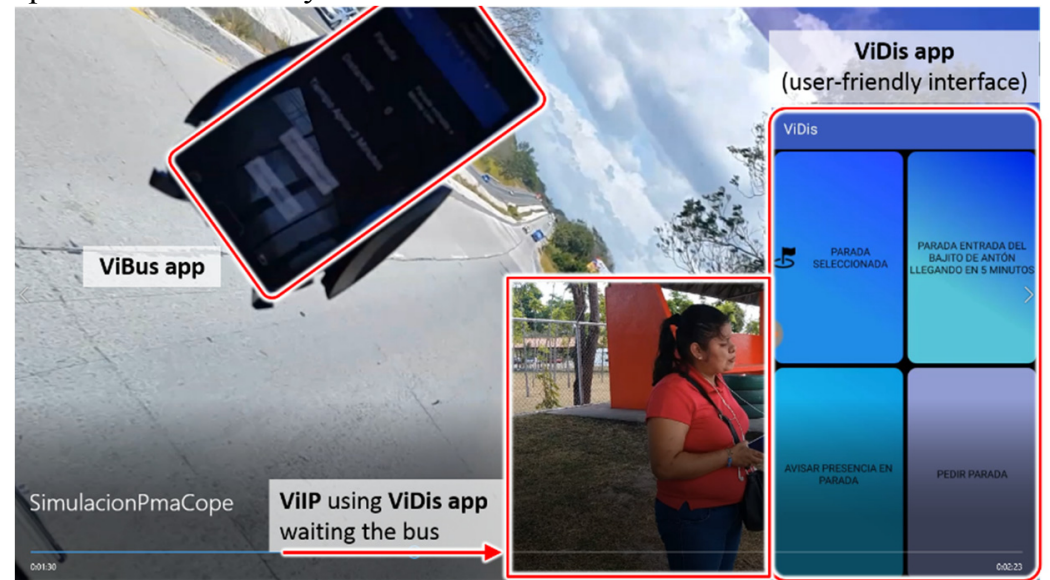

Figure 5. VilP waiting the bus using the ViDis app and the ViBus interface, during an experimental test.

\section{Conclusions and Future works}

In this paper has been outlined the conceptualization of the design and implementation of two systems ICT-based, addressed to provide helps to the mobility of visually impaired people in the public transportation in Panama. However, this proposal can be extended to other cities in the world, where technology not has been developed nowadays.

Several experiments have been carried out in outdoor, between bus stops in some transportation routes, given successful and promising results. It should be stressed that have been developed non-commercial prototypes, but they can be innovative products in the future.

Two people with full visually impaired have collaborated with our research group using the ViDis app and the MOVI-ETA module, and they have had a rapid learning using the prototypes and have expressed eagerness.

In future works, the prototypes will be improved, adding new characteristics and broaden their action fields to other outdoor and indoor zones.

\section{Acknowledgements}

The authors thank the funding of the Secretariat National of Science, Technology and innovation of Panama (SENACYT) within the framework 
of the project MOVIDIS with the contract by merit no. 109-2015-4FID14-073. Hector Montes and Yessica Saez also thank National Research System of Panama (SNI-SENACYT) by the support to their members.

\section{References}

1. WHO, 10 facts about blindness and visual impairment. (August 2014). Available in: http://www.who.int/features/factfiles/blindness/en/.

2. WHO, Vision impairment and blindness. (October 2017). Available in: http://www.who.int/mediacentre/factsheets/fs282/en/.

3. J.A. Muñoz, Las TIC y la discapacidad visual. Repositorio Universidad de la Coruña, Spain, 293 (2012).

4. E. Fürst, Mobility barriers in urban transport for the sight or hearing impaired: Solutions help all passengers. Real Corp 2010: Cities for Everyone. Liveable, Healthy, Prosperous. Vienna, 437 (2010).

5. E. Fürst and C. Vogelauer, Best and bad practices in public transport: approaches to a barrier-free design for the visually and hearing impaired. Proc. European Transport Conference 2013, Frankfurt, (2013)

6. G. Baudoin, O. Venard, G. Uzan, A. Rousseau, Y. Benabou, A. Paumier, J. Cesbron, The RAMPE Project: Interactive, Auditive Information System for the Mobility of Blind People in Public Transports. Proc. the 5th international conference on ITS Telecommunications ITST 2005, Brest France, (2005).

7. S. Azenkot, S. Prasain, A. Borning, E. Fortuna, R.E. Ladner, and J.O. Wobbrock, Enhancing independence and safety for blind and deaf-blind public transit riders. Proc. of the SIGCHI conference on Human Factors in computing systems. Vancouver, Canada, 3247 (2011).

8. M. Markiewicz and M. Skomorowski, Public transport information system for visually impaired and blind people. Transport Systems Telematics, 271 (2011).

9. F. Domínguez-Morrondo, Desarrollo de un sistema de guiado para invidentes. IMSERSO, Spain, (2005).

10. J.A. Muñoz and C. Blocona, Global Positioning System (GPS) accessibility for people with visual disability: the HaptiMap Project. Integración. Revista sobre discapacidad visual 61, 30 (2011).

11. H. Montes, I. Chang, G. Carballeda, J. Muñoz, A. García, R. Vejarano and M. Armada, Proc. RoboCity16 Open Conference on Future Trends in Robotics, C26, 211 (2016).

12. G. Carballeda, A. Arcia, R. Pérez, and H. Montes, Aplicación de Tecnologías de la Inf. y Com. para mejorar la Accesibilidad, 1, 93 (2016).

13. A. García, Y. Sáez, J. Muñoz, I. Chang, H. Montes, Proc. Sixth Eng., Science and Tech. Conf. "Trends and Challenges in Eng., Sci. and Tech., 821 (2017).

14. ITU. Radio Regulations Articles, (2012). 